\title{
Nonlinear Mechanical Response and Rippling of Thick Multiwalled Carbon Nanotubes
}

\author{
M. Arroyo* and T. Belytschko \\ Department of Mechanical Engineering, Northwestern University, Evanston, Illinois 60208, USA
}

(Received 5 May 2003; published 21 November 2003)

\begin{abstract}
The measured drop of the effective bending stiffness of multiwalled carbon nanotubes (MWCNTs) with increasing diameter is investigated by a generalized local quasicontinuum method. The previous hypothesis that this reduction is due to a rippling mode is confirmed by the calculations. The observed ripples result from a complex three-dimensional deformation similar to the Yoshimura buckling pattern. It is found that thick MWCNTs exhibit a well-defined nonlinear moment-curvature relation, even for small deformations, governed by the interplay of strain energy relaxation and intertube interactions. Rippling deformations are also predicted for MWCNTs subject to torsion, resulting in an effective torsional modulus much smaller than that predicted by linear elasticity.
\end{abstract}

DOI: 10.1103/PhysRevLett.91.215505

PACS numbers: $62.25 .+\mathrm{g}, 02.70 . \mathrm{Dh}, 46.32 .+\mathrm{x}, 61.46 .+\mathrm{w}$

A number of experiments have suggested that multiwalled carbon nanotubes (MWCNTs) can be used as basic elements of nanoelectromechanical systems (NEMS). In particular, nano-oscillators and actuators with MWCNTs in bending [1] and torsion [2,3] have been experimentally studied. In [1], the resonant frequencies of cantilevered MWCNTs excited electrically are reported. From these frequencies, the effective Young's modulus for the tubes was obtained by linear elasticity formulas. It was observed that, while small tubes exhibited a modulus of around $1 \mathrm{TPa}$, larger tubes were much more compliant with a modulus around $0.1 \mathrm{TPa}$. This dramatic reduction of the stiffness was attributed to the so-called rippling effect. Transmission electron micrographs (TEM) of bent thick MWCNTs were reported, in which a nonuniform deformation mode consisting of nearly periodic wavelike distortions in the compressed section of the tube was observed. A similar rippling deformation has been observed in other experiments $[4,5]$. Rippling is generally associated with elastic, reversible deformations $[1,6]$. The apparent contradiction between the widespread analysis of the mechanics of CNTs by linear elasticity, and complex nonlinear elastic phenomena such as rippling has been noted in [6]. In [6,7], the observations of [1] were analyzed qualitatively by $2 \mathrm{D}$ finite element models of anisotropic elasticity which developed rippling deformations.

In this Letter, we characterize the three-dimensional structure of rippling deformations and the associated energetics, by calculations of thick MWCNTs of realistic dimensions. The complex deformation morphologies we obtain, consistent with the experimental observations, are related to the Yoshimura pattern. We rationalize the emergence of rippling by the inextensional character of this pattern. The energetics of the rippling deformation mode depend on an interplay of the relaxation of the strain energy through the geometric instability and the van der Waals energy that keeps the ripples in place. This results in a substantially reduced effective modulus that changes with deformation. We predict an analogous rippling behavior for MWCNTs subject to torsion. To overcome the prohibitive number of degrees of freedom, the atomistic models were coarse grained by a method based on the finite element implementation of a recently proposed finite deformation continuum theory for crystalline monolayers [8]. In this method, a generalization of the local quasicontinuum method [9-11] to curved crystalline sheets, the continuum constitutive law is explicitly constructed in terms of the interatomic potential. This nonstandard continuum theory reproduces both the small and large deformation mechanical response of atomistic models of CNTs [12]. In the simulations below, Brenner's potential [13] has been used for the bonded interactions, while a Lennard-Jones potential models the van der Waals interactions, with the parameter set proposed in [14]. These nonbonded interactions are the only interlayer mechanical coupling in our simulations. Although it has been suggested that stronger coupling may occur [2], there is solid evidence of a weak interaction with very low sliding resistance like the one we adopt $[3,15,16]$. Equilibrium configurations are obtained by minimization of the total energy, i.e., the strain (bonded) energy plus the van der Waals energy.

A 34-walled $(5,5), \ldots,(170-170)$ nanotube about $23 \mathrm{~nm}$ in diameter and $124 \mathrm{~nm}$ in length is considered, similar to the nanotube reported in [4]. This system consists of about $5.9 \times 10^{6}$ atoms, that is $17.6 \times 10^{6}$ degrees of freedom. The finite element model used in these calculations has 300000 degrees of freedom, which is 60 times less than the atomistic system. By considering several finite element meshes, it is concluded that this model provides a "converged" solution (the errors introduced by the finite element approximation are comparable to the minimization tolerances). The rippled nanotube reported in [1] is thicker, with a diameter of $31 \mathrm{~nm}$ (about 45 walls). The simulation is performed as 
follows. First, the structure is fully relaxed. Then, it is bent with uniform curvature (the cylinder is mapped into a section of a torus). The radius of curvature is $165 \mathrm{~nm}$. Finally, the ends are fixed and the structure is relaxed [17].

Figure 1(a) shows a longitudinal cross section of the equilibrium configuration. This image is the computational analog of the TEM slices of rippled thick nanotubes reported in the literature [1,4]. The simulations reproduce very well the general features of the observed rippled nanotubes: nearly periodic wavelike distortions, whose amplitudes vanish for the inner tubes and smoothly increase towards the outer layer. The rippled MWCNT reported in [4] is subjected to a nonuniform curvature, which results in sharper ripples in the more severely bent portion. In the absence of detailed information about the loading in the experiments, the nanotubes are subjected to uniform bending in the simulations. For this reason, the periodicity of the simulated ripples is very regular, as in the observations in [1]. The wavelength of the ripples is well predicted (around $10 \mathrm{~nm}$ ).

The calculations provide information not available from the experiments. A three-dimensional image of the deformation is presented in Fig. 1(c). This image reveals a complex structure impossible to infer from the TEM micrographs, with an intercalation of several families of buckles tilted with respect to each other. Three characteristic cross sections are depicted in Fig. 1(b), which in principle could be obtained by TEM. These provide a new perspective on the deformation for they show that the structure consists of flat-top sections and

(a)

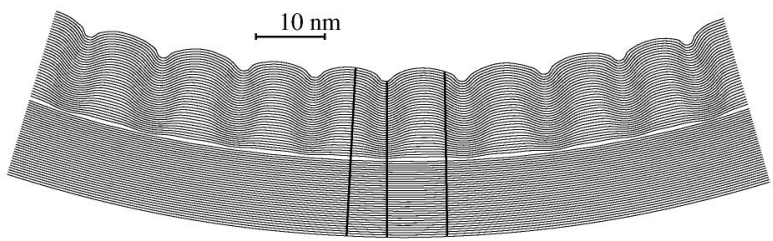

(b)
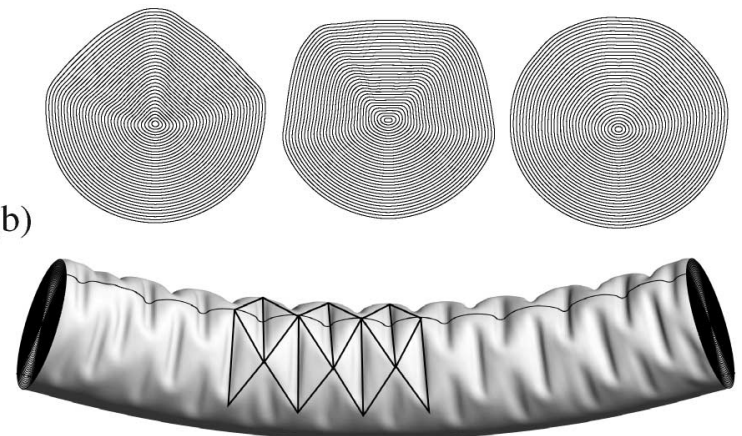

(c)

FIG. 1. Rippling of a 34-walled carbon nanotube: (a) longitudinal section of the central part of the simulated nanotube and (b) cross sections. The cross sections are marked as thick lines in (a). (c) Computed deformed configuration. The morphology of the ripples is reminiscent of the Yoshimura pattern. The ridges and furrows have been highlighted, as well as the trace of the longitudinal section. wedgelike sections, separated by nearly circular sections. The calculations show that the rippling deformation closely resembles the Yoshimura pattern or diamond buckling pattern [18], which characterizes the postbuckling behavior of cylindrical elastic shells.

Early work on the mechanics of carbon nanotubes identified the similarity of atomistic calculations to the linear buckling response of cylindrical elastic shells [19]. Linearized stability analysis predicts the so-called square pattern of Fourier buckling modes at the onset of buckling. Our simulations suggest that one can go further in this analogy with the classical theory of buckling of elastic shells. Indeed, the analysis of the postbuckling regime shows that the Fourier modes interact, giving rise to the diamond pattern [18]. As pointed out in [18], the Yoshimura pattern has the interesting geometric property of being a nearly isometric mapping of the undeformed surface, at the expense of creating sharp ridges and furrows. For a material like graphene, relatively compliant to bending but very stiff to in-plane deformation, this is a very advantageous deformation mechanism, which releases much of the membrane strain energy at the cost of some flexural energy. Indeed, our calculations indicate that rippled MWCNTs have a significantly lower strain energy than uniformly bent MWCNTs, and that the distribution of the strain energy into membrane and bending energy also differs in the two cases; while for uniformly bent tubes nearly $100 \%$ of the strain energy is membrane energy, rippling increases the bending energy to $20 \%$ of the strain energy. Obviously, rippling cannot release the membrane energy in the part of the MWCNT subject to tension, e.g., the lower part in Fig. 1(a).

This very regular buckling pattern seems to be a distinctive feature of thick multiwalled nanotubes without internal hollow space, i.e., those in which the innermost nested tube radius is of the order of the interlayer spacing. Experimental observations and atomistic calculations suggest that bent single-walled and multiwalled nanotubes with internal hollow space display sharp kinks, where most of the deformation is localized in a small region [16,20]. In MWCNTs without internal hollow space, the outer walls are constrained by the inner tubes, and deep buckles do not have space to develop. Instead, these systems choose the Yoshimura mechanism to release in a very distributed fashion the compressive membrane strain energy.

Precise information about the energetics of the rippling deformation can be extracted from the calculations. Figure 2(a) shows the energy of the system as a function of the bending curvature for the 34-walled CNT. Linear elasticity predicts a quadratic growth of the strain energy with respect to curvature. For illustration, the strain energy of the uniformly bent MWCNT is plotted in Fig. 2(a) for several radii of curvature $R$, and indeed the graph is perfectly fitted by $E_{\text {strain }} \propto(1 / R)^{2}$. For such a uniform bending the van der Waals energy is nearly insensitive to deformation, and therefore the total energy 

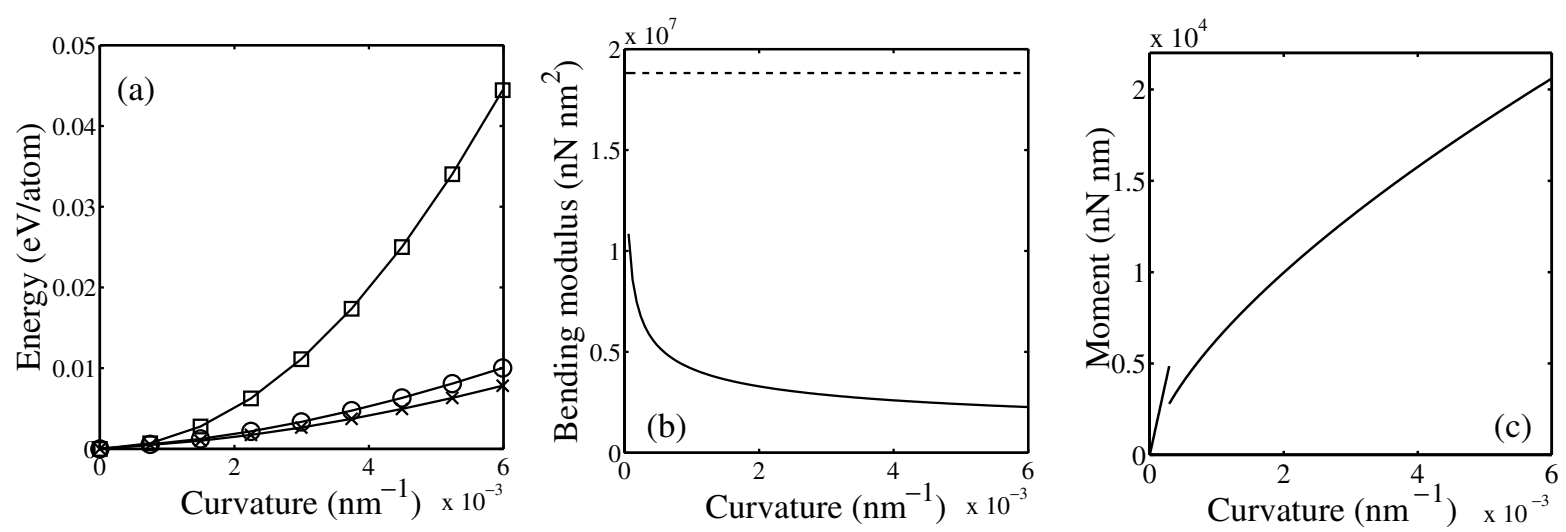

FIG. 2. Bent 34-walled CNT. (a) Energy change relative to straight relaxed configuration vs curvature; strain energy ( $\square$ ) for uniform bending (ideal); total energy $(\bigcirc)$ and strain energy $(X)$ for rippled deformation (actual). (b) Variation of the effective bending modulus with curvature (solid line) and linear elasticity modulus (dashed line). (c) Bending moment vs curvature: initial linear regime for small curvatures, followed by the nonlinear response characteristic of rippling.

also follows the quadratic law. However, the actual behavior of the system greatly deviates from this ideal linearly elastic response, as can be observed in the figure. The mechanical relaxation through the rippling deformation leads to much lower values of strain energy of the system. The figure also shows that the highly inhomogeneous deformation of rippling leads to an increase in van der Waals energy with deformation (the $\circ$ and the $\times$ curves deviate). The global response of the system is dictated by the evolution of the total energy, which in this range is very accurately fitted by $E_{\text {tot }} \propto(1 / R)^{a}$ with $a=1.66$.

This response differs from that predicted by atomistic simulations of SWCNTs or small hollow MWCNTs, with an initial nearly quadratic growth of the energy with respect to the deformation $(a=2)$, followed by a postbuckling regime characterized by a linear growth $(a=1)$, which implies that a constant force is sufficient to further deform the tube, and that the effective stiffness is reduced to nearly zero $[8,19,20]$. These two regimes are adequately explained by linear elasticity and linearized stability analysis [19]. These theories, however, do not explain the observed well-defined stiffened postbuckling behavior $(1<a<2)$, even if the van der Waals interactions are included in the analysis, as in [21]. For the thick MWCNT under consideration, the outer shells are very prone to structural instabilities, even for small bending curvatures. Consequently, the initial quadratic regime is practically absent, and the overall response is dictated by the nonlinear mechanics of rippling.

These observations explain the reduction of the effective modulus for thick MWCNTs. Based on the fit to the total energy described above, Fig. 2(b) shows the bending modulus of the 34-walled CNT as a function of curvature. The usual beam theory definition of the bending modulus (second derivative of the elastic energy per unit length with respect to the curvature) is adopted. Since $a<2$, the effective tangent bending modulus monotonically decreases as $\propto(1 / R)^{a-2}$. This formula is not valid as $1 / R \rightarrow 0$, since the quadratic regime holds in a small neighborhood of the straight configuration, for radii of curvature larger than about $3000 \mathrm{~nm}$. It can be observed in the figure that this modulus changes with deformation, in contrast with linear theory. In particular, it is found that the effective bending modulus of the MWCNT for the largest curvature considered $(1 / R=$ $\left.1 / 165 \mathrm{~nm}^{-1}\right)$ is 8.3 times smaller than the linear elastic modulus. For an intermediate curvature $(1 / R=$ $\left.1 / 330 \mathrm{~nm}^{-1}\right)$, the linear elastic modulus is reduced by a factor of 6.6. Figure 2(c) shows that the bending moment vs curvature relation obtained from the calculations is qualitatively different from that predicted for small CNTs [19]. An additional set of calculations for a smaller 20walled $(5,5), \ldots,(100,100)$ CNT leads to a very similar

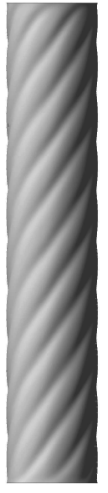

(a)

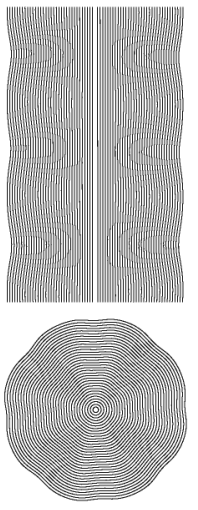

(b)

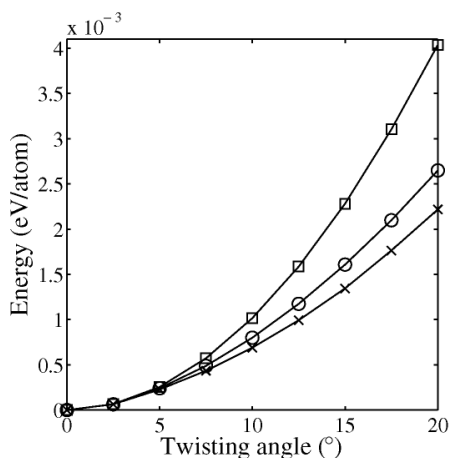

(c)
FIG. 3. Twisted 34-walled CNT $124 \mathrm{~nm}$ long. (a) Deformed configuration for the last computed deformation $\left(\gamma_{\text {out }}=3.3 \%\right.$ ). (b) Longitudinal and cross sections. (c) Energy change relative to straight relaxed configuration; strain energy ( $\square$ ) for uniform twisting (ideal); total energy $(\bigcirc)$; and strain energy $(X)$ for rippled deformation (actual). 
behavior, although in this case $a=1.58$ very accurately describes the computed values of the total energy.

Motivated by nano-oscillators and actuators in which thick MWCNTs act as torsional springs [2,3], we investigate whether an inhomogeneous deformation analogous to rippling develops in torsion. The 34-walled CNT $124 \mathrm{~nm}$ long is considered. In [12] we showed that small CNTs subject to torsional deformations conform with linear elasticity predictions up to relatively large deformations [a twisted $(10,10)$ CNT buckled for a shear strain of $\gamma=5 \%$ ]. For a uniformly twisted MWCNT of length $L$, the shear strain for the $i$ th layer can be computed as $\gamma_{i}=\Theta r_{i} / L$, where $\Theta$ is the twisting angle and $r_{i}$ is the radius of the $i$ th tube. The highest shear strain is that of the outer tube $\gamma_{\text {out }}$. The linear elastic torsional spring constant of such a tube follows $\kappa=\left(2 \pi G_{s} / L\right) \sum_{i} r_{i}^{3}=$ $6.2 \times 10^{4} \mathrm{aJ}$, where $G_{s}$ is the surface shear modulus $\left(83.5 \mathrm{~J} / \mathrm{m}^{2}\right.$ for Brenner's potential [12]), and the sum runs over the 34 individual CNTs [22]. This leads to $E_{\text {strain }}=1 / 2 \kappa \Theta^{2}$. This energetic prediction perfectly matches calculations in which the layers of the twisted 34-walled CNT are constrained to remain cylindrical, i.e., not allowed to buckle, shown in Fig. 3(c). However, the unconstrained system develops a nonuniform deformation far more efficient from the energetic point of view, as shown in Fig. 3. Torsional rippling develops at a relatively small shear strain of $\gamma_{\text {out }}=0.8 \%$. The van der Waals energy significantly increases with deformation, resulting in a stiffened postbuckling behavior. For these calculations, the energy growth very accurately follows $E_{\text {tot }} \propto$ $\Theta^{a}$ with $a=1.73$ in the postbuckling regime $\left(\Theta \geq 5^{\circ}\right)$. Our simulations suggest that torsional rippling does not reduce the rigidity of MWCNTs as much as rippling in bending [compare Fig. 3(c) with Fig. 2(a)]; the exponent $a$ is closer to 2 in torsion. The experiments on torsional oscillators in [2,3] involve quite thick MWCNTs, and rough estimates from the data provided seem to indicate that the deformations in the experiments would cause torsional rippling to occur. Although to the best of our knowledge torsional rippling has never been described in the literature, we believe that there is experimental evidence of it (see Fig. 37 in [23]) [24].

In summary, we have shown that thick MWCNTs are very prone to develop rippling deformations in bending and twisting. For these structures, the linear regime is practically absent, or restricted to a small range of deformations. Instead, the response is dominated by nearly inextensional rippling deformation modes. Rippling leads to an overall nonlinear response characterized by $E_{\text {tot }} \propto$ [deformation] ${ }^{a}$, with $1<a<2$. The parameter $a$ depends on the size [25] and the deformation mode. Linear elasticity predictions $(a=2)$ and linearized buckling analysis $(a=1)$ should be used with caution for these systems.

We gratefully acknowledge the grant support from the NASA University Research, Engineering and Technology Institute on Bio Inspired Materials (BIMat).
*Now at the Division of Engineering and Applied Science, California Institute of Technology, Pasadena, CA 91125, USA.

[1] P. Poncharal, Z. Wang, D. Ugarte, and W. de Heer, Science 283, 1513 (1999).

[2] P. A. Williams, S. J. Papadakis, A. M. Patel, M. R. Falvo, S. Washburn, and R. Superfine, Phys. Rev. Lett. 89, 255502 (2002).

[3] A. M. Fennimore, T. D. Yuzvinsky, W. Q. Han, M. S. Fuhrer, J. Cumings, and A. Zettl, Nature (London) 424, 408 (2003).

[4] T. Kuzumaki, T. Hayashi, H. Ichinose, K. Miyazawa, K. Ito, and Y. Ishida, Philos. Mag. A 77, 1461 (1998).

[5] O. Lourie, D. M. Cox, and H. D. Wagner, Phys. Rev. Lett. 81, 1638 (1998).

[6] J. Z. Liu, Q. Zheng, and Q. Jiang, Phys. Rev. Lett. 86, 4843 (2001).

[7] J. Z. Liu, Q. Zheng, and Q. Jiang, Phys. Rev. B 67, 075414 (2003).

[8] M. Arroyo and T. Belytschko, J. Mech. Phys. Solids 50, 1941 (2002).

[9] E. B. Tadmor, M. Ortiz, and R. Phillips, Philos. Mag. A 73, 1529 (1996).

[10] E. B. Tadmor, G. S. Smith, N. Bernstein, and E. Kaxiras, Phys. Rev. B 59, 235 (1999).

[11] G. S. Smith, E. B. Tadmor, and E. Kaxiras, Phys. Rev. Lett. 84, 1260 (2000).

[12] M. Arroyo and T. Belytschko (to be published).

[13] D.W. Brenner, Phys. Rev. B 42, 9458 (1990).

[14] L. A. Girifalco, M. Hodak, and R. S. Lee, Phys. Rev. B 62, 13104 (2000).

[15] J. Cumings and A. Zettl, Science 289, 602 (2000).

[16] D. Qian, W. K. Liu, S. Subramoney, and R. S. Ruoff, J. Nanosci. Nanotechnol. 3, 185 (2003).

[17] Sufficiently long tubes have been considered, to restrict the boundary effects to a small region near the ends, while most of the tube displays uniform rippling.

[18] Z. Bažant and L. Cedolin, Stability of Structures (Oxford University Press, New York, 1991).

[19] B. I. Yakobson, C. J. Brabec, and J. Bernholc, Phys. Rev. Lett. 76, 2511 (1996).

[20] S. Iijima, C. J. Brabec, A. Maiti, and J. Bernholc, J. Chem. Phys. 104, 2089 (1996).

[21] C. Q. Ru, Phys. Rev. B 62, 16962 (2000).

[22] The formula used in [2] $\bar{\kappa}=\pi r_{\text {out }}^{4} G /(2 L)$, where $G=$ $G_{s} / t$ is a homonenized shear modulus and $t=0.34 \mathrm{~nm}$ is the interlayer spacing, provides a reasonable approximation, $\bar{\kappa}=6.0 \times 10^{4}$ aJ.

[23] L. Forró and C. Schönenberger, Top. Appl. Phys. 80, 329 (2001).

[24] The authors in [23] associate the pattern in their AFM image with rippling in bending; however, the morphological agreement with our simulations suggests that it is in fact an instance of torsional rippling.

[25] Additional calculations on a series of MWCNTs of diameters ranging from 10 to $24 \mathrm{~nm}$ subject to torsion lead to values of $a$ around 1.7 , and suggest that $a$ increases with the diameter in this range, maybe due to the fact that fewer walls in van der Waals contact lead to a less constrained system. 